standard) of SOC was $91.7 \%$ and $92.9 \%$ respectively while GXP was 50.8\% and 99.2\%, respectively. LAM, when used with SOC, did not improve sensitivity or specificity in any CD4 strata, however when used with GXP increased sensitivity from $20 \%$ to $50 \%$ at CD $4<50$. There was a marginally significant difference $(p=0.08)$ at 14 -day TB treatment initiation between the GXP and SOC phases but no difference at 28-days. Among those initiating therapy, the median time to TB treatment initiation was shorter for the GXP arm (4 vs15 days).

Conclusions GXP did not significantly increase the number or accuracy of TB diagnoses compared to SOC but reduced median number of days to TB treatment by 11 days. GXP and LAM when used together have the potential to rapidly identify TB in patients with advanced HIV disease.

\section{OA-005 PERFORMANCE OF XPERT MTB/RIF AND DETERMINE LAM IN HIV-INFECTED ADULTS IN PERI-URBAN SITES IN ZAMBIA (CDC OP-X STUDY)}

Margaret Phiri Kasaro, ${ }^{1}$ Brenda Muluka, ${ }^{1}$ Kaunda Kaunda, ${ }_{1}^{1}$ Jill Morse, Andrew Westfall, ${ }^{2}$ Nathan Kapata, ${ }^{3}$ Annika Kruuner, ${ }^{2}$ German Henostroza, ${ }^{2}$ Stewart Reid2. ${ }^{1}$ CIDRZ, Zambia; ${ }^{2}$ UAB, United States of America; ${ }^{3}$ Ministry of Health, Zambia

\subsection{6/bmjgh-2016-000260.14}

Background Tuberculosis (TB) mortality in HIV-infected patients remains high in sub-Saharan Africa. Inadequate diagnostic tools delay time to TB treatment.

Methods A two-phase TB diagnostic study was conducted among HIV-infected adult patients from 2014-2016. Patients underwent history/physical exam, chest x-ray, urine for lipoarabinomannan (LAM), sputum smear and culture. We evaluated sensitivity, specificity and time to appropriate treatment within 14 and 28 days of screening for culture-positive patients, comparing Xpert MTB/RIF assay (GXP), and LAM to standard-of-care (SOC) in 3 peri-urban clinics. chi-square and Wilcoxon Rank-Sum tests were used to test for differences between SOC and GXP for categorical variables and continuous variables, respectively.

Results 1353 patients were enrolled; 755 in the SOC arm and 598 in the GXP arm. Median age was 34.3 and $65.1 \%$ were male. TB was diagnosed by any method (smear, clinical, GXP, LAM, culture) in 237 (17.5\%) and with positive MTB culture in 152 (11.2\%); 84 and 68 in the SOC and GXP arms, respectively. The overall sensitivity and specificity (culture as reference 